Curr Opin Hematol. 2020 January ; 27(1): 11-17. doi:10.1097/MOH.0000000000000554.

\title{
Family Studies of WHIM Syndrome
}

\author{
David C. Dale ${ }^{1}$, Emily Dick ${ }^{2}$, Merideth Kelley ${ }^{1}$, Vahagn Makaryan ${ }^{1}$, Jim Connelly ${ }^{3}$, Audrey \\ Anna Bolyard ${ }^{2}$ \\ ${ }^{1}$ University of Washington, Department of Medicine, Seattle, WA \\ ${ }^{2}$ University of Washington, Severe Chronic Neutropenia International Registry, Seattle, WA \\ ${ }^{3}$ Vanderbilt University, Department of Pediatrics, Nashville, TN
}

\begin{abstract}
Purpose of the review-WHIM syndrome (warts, hypogammaglobulinemia, immunodeficiency, myelokathexis, or WHIMs) is a very rare autosomal dominant immunodeficiency disorder attributable to mutations in $C X C R 4$. We reviewed clinical manifestations in 24 patients in 9 families to expand understanding of this syndrome.
\end{abstract}

Recent findings-Warts, cellulitis and respiratory infections are common in patients with WHIMs. Less commonly these patients have congenital heart disease, HPV associated malignancies and lymphomas. Hearing loss due to recurrent otitis media is another important complication. Treatment with granulocyte colony-stimulating factor is controversial; this review indicates that it is effective to prevent and treat infections based upon long-term observations of patients enrolled in the Severe Chronic Neutropenia International Registry. Understanding the natural history and diversity of this syndrome are important for ongoing clinical trials of novel agents to treat WHIMs.

Summary-WHIM syndrome has diverse manifestations; some features occur consistently in almost all patients, e.g., neutropenia, lymphocytopenia and mild hypogammaglobulinemia. However, the clinical consequences are quite variable across patient cohorts and within families. Each complication is important as a cause for morbidity and a source for patient and family concerns.

\section{Keywords}

WHIM syndrome; $C X C R 4$; neutropenia; lymphocytopenia; HPV associated malignancies; deafness

\section{Background}

WHIM syndrome (warts, hypogammaglobulinemia, immunodeficiency, myelokathexis, or WHIMs) is a very rare autosomal dominant immunodeficiency disorder attributable to mutations in CXCR4. Although it is very rare, there are more than 100 published case reports [1] since the original reports by Zeulzer et al and Crill [2**, $\left.3^{*}\right]$ in 1964. In 2003,

CORRESPONDING AUTHOR: David C. Dale, MD, University of Washington, Box 356422, 1959 NE Pacific St., Rm AA522, Seattle, WA 98195, Tel: 206-543-7215, Fax: 206-685-4458, dcdale@uw.edu. 
Hernandez et al reported that WHIMs is cause by mutations in $C X C R 4$. [4**] Several papers now describe the clinical consequences of these mutations. [5-11**] The severity of the leukopenia (decreased neutrophils, lymphocytes and monocytes) is a unique feature of this immunodeficiency syndrome. This finding alone should readily lead clinicians to consider this diagnosis. Leukopenia occurs because all types of leukocytes express CXCR4. The $C X C R 4$ mutations cause truncation of the receptor protein which impairs receptor internalization when leukocytes are exposed to their natural ligand, CXCL12. This chemokine was originally called stromal cell derived factor-1 (SDS-1). [2**, 12**] This defect in receptor function results in abnormal retention of leukocytes in bone marrow and at other extravascular sites. The bone marrow shows accumulation of neutrophils at various stages of apoptotic death marked by shrinkage of cells with extremely pyknotic nuclei. [2**, 13] WHIMs is currently of special interest because of the finding that $C X C R 4$ antagonists can increase blood leukocytes and may be an effective therapeutic option for these patients. [14-18**]

WHIMs is one of the diverse causes for severe chronic neutropenia being investigated through the Severe Chronic Neutropenia International Registry. (SCNIR, https:// depts.washington.edu, 19) Understanding the natural history of WHIMs is critical for planning clinical trials and evaluating treatment outcomes. It is also important for trying to answer the simple question that patients often ask, "Doctor, what will happen to me?" For this report we reviewed previous studies of WHIMs patients and families and family histories and clinical data for 9 families with WHIMs enrolled in the SCNIR, with particular attention to effectiveness of treatment with granulocyte colony-stimulating factor (G-CSF) treatment.

\section{Severe Chronic Neutropenia International Registry (SCNIR)}

The Severe Chronic Neutropenia International Registry (SCNIR) was established in 1994 to study the clinical consequences and treatment of severe chronic neutropenia. The Registry has proven to be an extremely useful resource for understanding the clinical consequences of chronic neutropenia and for collaborations to define its genetic and molecular causes, including discovery of ELANE, HAX1, G6PC3, TCIRG1, and CLPB mutations as causes of severe chronic neutropenia. Patients with WHIM syndrome were included because of the severity of associated neutropenia. Before it was possible to make a genetic diagnosis, patients were often given the clinical diagnosis of myelokathexis based on the characteristic findings of severe neutropenia and lymphocytopenia and the abnormal morphology of the neutrophils, particularly shrinkage of nuclear chromatin in marrow neutrophils.

\section{Methods}

Through the SCNIR we identified 9 WHIMS families in the US with 24 affected members: 13 female, 11 male. The $C X C R 4$ mutations were: R334X (16), E343X (3), fs $344 \mathrm{X}$ (3), fs $365 X$ (2), inferring the mutation in some individuals from sequence results for the index patient in the family.

The pedigrees for the 9 families are shown in Figure 1. 


\section{Family Vignettes}

Family A. Mutation R334X-The daughter of two unaffected parents had severe neutropenia recognized at 6 years of age. She had two unaffected siblings. At diagnosis, her mean blood neutrophil count (ANC) was $0.600 \times 10^{9} / \mathrm{L}$ (range 0.000 to $4.480 \times 10^{9} / \mathrm{L}$ ). Low dose, subcutaneous G-CSF treatment was initiated. After 6.4 years of G-CSF treatment, at age 10.6 years, she was diagnosed as having myelodysplasia. Her spleen was removed at 11.4 years. Soon thereafter diagnosis was changed to acute myeloid leukemia (AML), and she received an allogeneic hematopoietic stem cell transplant (HSCT) at 11.5 years. The patient relapsed and died at 12.3 years.

Family B. Mutation fs365X-In this family there was an affected mother and daughter, with other unaffected family members. The affected mother was found to be neutropenic at 9 weeks of age, mean ANC was $0.086 \times 10^{9} \Lambda$ (range 0.056 to $0.117 \times 10^{9} / \mathrm{L}$ ). G-CSF treatment was initiated at 7.6 years of age and administration continues to current age of 36.7 , i.e., for 29 years. Her daughter, neutropenic at birth, mean ANC was $0.953 \times 10^{9} / \mathrm{L}$ (range 0.066 to $3.120 \times 10^{9} / \mathrm{L}$ ). G-CSF treatment started at 3.5 weeks of age. After 4.2 years of G-CSF, she received HSCT because of her severe neutropenia and thrombocytopenia. Currently she is doing well at age 10.3.

Family C. Mutation E343X-There are affected patients in three generations in this family: an affected mother with affected son and grandson. The affected mother had multiple documented pneumonias and other illnesses in childhood and has consistently selfadministered G-CSF for several years. Her son with baseline ANC of $0.205 \times 10^{9} / \mathrm{L}$ (range 0.000 to $0.550 \times 10^{9} / \mathrm{L}$ ), administers G-CSF sporadically. Her grandson, diagnosed at birth, mean ANC was $0.058 \times 10^{9} / \mathrm{L}$ (range 0.026 to $0.140 \times 10^{9} / \mathrm{L}$ ). G-CSF treatment started at 12 weeks of age and has been consistently administered for 3 years.

Family D. Mutation R334X-There are affected individuals in four generations in this family: affected father, daughter, granddaughter, great grandson with many other unaffected family members. The father was diagnosed with WHIMs at age 45 years; his mean ANC was $0.370 \times 10^{9} / \mathrm{L}$ (range 0.090 to $1.248 \times 10^{9} / \mathrm{L}$ ). He began G-CSF at 74.3 years and continued for 7.5 years. His daughter, diagnosed early in life, had mean ANC was $0.309 \times$ $10^{9} / \mathrm{L}$ (range 0.200 to $0.430 \times 10^{9} / \mathrm{L}$ ); G-CSF administration was inconsistent. She had multiple warts on her hands and heaping warts on plantar aspect of feet. Her warts were far worse than any other patients we have seen. At age 33.7 years she developed HPV positive invasive vulvar carcinoma and died at age 46.2 years, after many surgical procedures to remove the invasive cancer. In the third generation, the granddaughter was diagnosed at birth with the finding of mean ANC was $0.179 \times 10^{9} / \mathrm{L}$ (range 0.090 to $0.308 \times 10^{9} / \mathrm{L}$ ), G-CSF treatment started at 10 years of age and continued inconsistently for 11.3 years to current age of 21.4 years. Recently she was given prophylactic G-CSF at the time of an elective cholecystectomy and did remarkably well with no infectious complications. Her son, an affected member in the fourth-generation of this family, was also diagnosed at birth with neutropenia (mean ANC was $0.034 \times 10^{9} / \mathrm{L}$ (range 0.032 to $0.036 \times 10^{9} / \mathrm{L}$ ), patent ductus arteriosus, multiple skin tags with cartilaginous continuity, and a dilated kidney. G-CSF treatment started at 17 weeks and continues to current age of 21 weeks. 
Family E. Mutation R334X-Two generations are known to be affected in this family: an affected mother and son, with other unaffected family members. The mother, age 40 years, mean ANC $0.292 \times 10^{9} / \mathrm{L}$ (range 0.165 to $0.514 \times 10^{9} / \mathrm{L}$ ), has had few minimal infections but significant hearing loss attributable to recurrent otitis media. She has had only short-term G-CSF. Her son, diagnosed at birth (mean ANC $0.129 \times 10^{9} / \mathrm{L}$, range 0.000 to $0.656 \times$ $10^{9} / \mathrm{L}$ ) started on G-CSF at 16 weeks old and continued for nearly 16 years. He has extensive molluscum contagiosum unimproved on G-CSF therapy.

Family F. Mutation in CXCR4 is R334X-Three generations are known to be affected in this family: an affected mother, 2 sons and daughter, a granddaughter and a grandson with other unaffected family members. The mother died at age 73 from colon cancer and was previously treated for cervical cancer. She had two sons. One son, mean ANC $0.63 \times 10^{9} / \mathrm{L}$ (range 0.12 to $1.5 \times 10^{9} / \mathrm{L}$ ), has had multiple deep tissue abscesses and hearing loss. He has received G-CSF intermittently. The other son also has been on intermittent G-CSF and developed non-Hodgkin lymphoma and died at age 48. One daughter mean ANC $1.232 \times 10$ ${ }^{9} / \mathrm{L}$ (range 0.246 to $2.645 \times 10^{9} / \mathrm{L}$ ), also has been on intermittent G-CSF. One granddaughter, mean ANC $0.586 \times 10^{9} / \mathrm{L}$ (range 0.000 to $6.478 \times 10^{9} / \mathrm{L}$ ), has multiple severe warts especially on hands and feet. She also has received sporadic G-CSF. Less is currently known about the other grandson.

Family G. Mutation R334X-This affected male was diagnosed with Tetralogy of Fallot with dysplastic pulmonary valve, ductus arteriosus at birth and neutropenia at 8 weeks of age. His mean ANC was $1.705 \times 10^{9} / \mathrm{L}$ (range 0.230 to $4.185 \times 10^{9} / \mathrm{L}$ ). He has been continuously treated with G-CSF for nearly 19 years, current age 20 years.

Family H. Mutation R334X-This is a two generation family, an affected mother and son, with other known unaffected family members. The mother, age 60.4 years, was diagnosed at 8.7 years of age, mean ANC $0.212 \times 10^{9} / \mathrm{L}$ (range 0.000 to $1.020 \times 10^{9} / \mathrm{L}$ ). She has a history multiple deep tissue abscesses. She initiated G-CSF at age 36, and she received intermittent G-CSF administration over the next 24 years usually as part of treatment for abscesses. On antibiotics without G-CSF the abscesses did not heal or did so very slowly. Her son, diagnosed at 3.9 years of age, mean ANC $1.162 \times 10^{9} / \mathrm{L}$ (range 0.000 to $4.680 \times 10^{9} / \mathrm{L}$ ), was initially treated with IVIG at age 10 to 13 . He had an anaphylactic reaction to the IVIG and it was not continued. G-CSF was initiated to treat infections at age 17 years and it was administered intermittently. He had appendicitis at age 25 , was not treated at that time with G-CSF and had a prolonged recovery. He has had multiple episodes of pneumonia, otitis and cellulitis, and he has severe hearing loss.

Family I. Mutation fs $344 X$ - This is a two generation family, an affected mother, daughter and son with other unaffected family members. The mother currently age 43.4 years was diagnosed with neutropenia at 8 months of age, mean ANC $0.384 \times 10^{9} / \mathrm{L}$ (range 0.048 to $2.276 \times 10^{9} / \mathrm{L}$ ). She also has mitral valve prolapse, tricuspid regurgitation, and a pelvic kidney. G-CSF was initiated at age 20 years for 6 weeks, but it was stopped when she learned she was pregnant. Her daughter, diagnosed with neutropenia also at age 8 months, mean ANC $0.091 \times 10^{9} / \mathrm{L}$ (range 0.000 to $0.200 \times 10^{9} / \mathrm{L}$ ) was initiated G-CSF at 9 months 
but it was stopped at age 2 because of thrombocytopenia. Her son, mean ANC $0.807 \times 10^{9} / \mathrm{L}$ (range 0.012 to $2.214 \times 10^{9} / \mathrm{L}$, was born with complex cardiac anomaly, pelvic kidney and malrotation of the intestines.

\section{Laboratory Data}

As in other reports, the cardinal hematological features for these 24 patients are leukopenia, neutropenia and lymphocytopenia. Before G-CSF and when the patients were relatively well, median leukocyte counts were: WBC $1.45 \times 10^{9} / \mathrm{L}$ (mean +/- SEM 1.80+/ $-0.22 \times 10^{9} / \mathrm{L}$ ), ANC $0.196 \times 10^{9} / \mathrm{L}$ (mean $0.41+/-0.08 \times 10^{9} / \mathrm{L}$ ), ALC $0.838 \times 10^{9} / \mathrm{L}$ (mean $1.16+/-0.2010^{9} / \mathrm{L}$ ). The family medical records and vignettes also reveal that these patients can increase their ANC to normal with infections or treatment with G-CSF but the lymphocytopenia persists. On treatment with G-CSF (usual doses of $1-3 \mathrm{mcg} / \mathrm{kg} / \mathrm{day}$ ) medians counts were: WBC $2.12 \times 10^{9} / \mathrm{L}$ (mean $3.144+/-0.50 \times 10^{9} / \mathrm{L}$ ), ANC $0.92 \times 10^{9} / \mathrm{L}$ (mean $2.07+/-0.46 \times 10^{9} / \mathrm{L}$ ) and ALC $0.56 \times 10^{9} / \mathrm{L}$ (mean $0.829+/-0.15 \times 10^{9} / \mathrm{L}$ ).

Previous reports indicate that most WHIM patients have moderately reduced, but not severely reduced immunoglobulin levels. For these patients results were similar. Mean and median values for these patients were: IgG mean $677+/-55.6$ (SEM), median 635; IgM mean 78 +/- 12.6, median 74; IgA mean $93+/-19.3$, median 58; all mg/dL.

\section{Clinical Observations}

These 9 families illustrate the diversity of clinical problems in patients with WHIM syndrome. As shown in figure 1, only 5 of the 24 patients had severe warts. Other patients had only a few warts and some described spontaneous regressions of their warts. Although warts are contagious, there was no consistent pattern of occurrence within families. One patient had the clinical diagnosis of molluscum contagiosum with a distinctly different pattern of warts occurring diffusely on the body previously not reported in WHIMs, although known to occur in immunodeficiency patients.

Congenital anomalies, particularly cardiac anomalies have been previously reported in WHIM syndrome. [10] As noted above, in 3 families, patients had congenital anomalies: Families D, G and I. Cervical cancer and lymphoma are known complications in WHIMs patients, and both were also observed in this study. In addition one patient developed AML, having been on low-dose G-CSF for more than 10 years.

Two patients received HSCT; one because of the development of AML; this patient died. The other patient received HSCT because of persistent neutropenia and thrombocytopenia and has done well. Our review of these complications shows no particular patterns within families, with distinctive clinical patterns for each individual rather than each family.

Hearing loss associated with large perforations of tympanic membranes appears to be a relatively frequent complication of WHIM syndrome. Otitis is a common problem for WHIMs patients, and 3 of 24 reported significant hearing loss. We do not know if audiograms would detect more patients with hearing impairment.

Curr Opin Hematol. Author manuscript; available in PMC 2021 January 01. 
It is difficult to compare accurately the frequency and severity of infection across published reports. For patients enrolled and followed prospectively through the SCNIR, our impression is that these patients have had fewer serious infections than in the other recent clinical papers on WHIMs. [7**, 11**] Whether this is attributable to greater prophylactic use of G-CSF or other specific factors is not readily explained. It is also possible that the US pedigrees are large and include patients with less severe WHIMs.

\section{Treatment with Granulocyte Colony-stimulating Factor (G-CSF)}

Under an agreement with the University of Washington, Amgen has provided G-CSF/ Neopogen to US patients with severe chronic neutropenia who are enrolled in the SCNIR in support of this long-term observational study. WHIMs are eligible to participate. The G-CSF is prescribed by the patient's personal physician and the patients and physicians are required to report treatment responses to the SCNIR on an annual basis, providing much of the data for this summary report.

For the 9 families, 21 of 24 are known to have received G-CSF, 7 continually and 14 intermittently. The usual doses for WHIMs are $1-3 \mathrm{mcg} / \mathrm{kg} / \mathrm{day}$; median dose for 21 patients in this report was 1.6 micrograms $/ \mathrm{kg} /$ day. Based on observations made through the SCNIR, G-CSF doses should be titrated to minimize symptoms and maintain an ANC between 1.0$2.0 \times 10^{9} / \mathrm{L}$. Higher counts are not necessary to prevent infections and are frequently associated with adverse effects, e.g., bone pain and headaches.

In our observations, daily, alternate-day, or 2-3 days per week administration of G-CSF to patients with frequent cellulitis, otitis, pharyngitis and respiratory symptoms as described above has consistently reduced the frequency and severity of these inflammatory events. Patients with WHIMs rarely have urinary tract or gastrointestinal infections, bacteremia, sepsis or infections requiring hospitalizations. For patients not receiving G-CSF to prevent infections, i.e. not treated prophylactically, G-CSF was often administered when infections occurred. Our uncontrolled observations suggest that it is of benefit, as exemplified in the following examples.

1. This 40.5 year old male (Family $\mathrm{H}$ ) regularly had environmental dust exposure and symptoms of early chronic obstructive pulmonary disease. He had appendicitis at age 25, was not treated with G-CSF and had a prolonged recovery. He was hospitalized on multiple occasions for bronchitis or pneumonia. His physician and the patient consistently reported benefit from GCSF administration to recover from his infections.

2. This 60.4 year old female (Family $\mathrm{H}$ ) has had recurrent deep skin abscesses occurring spontaneously and responding very slowly to antibiotics. Addition of G-CSF to antibiotic treatment accelerated her recovery on multiple occasions.

3. This 21.4 year old female (Family D) developed gallstones and required an elective cholecystectomy. During the perioperative period, her physicians elected to treat her with G-CSF and she went through the postoperative period with no complications. 
One patient developed AML after approximately 10 years of G-CSF therapy. AML has not been previously described in WHIMs patients, and whether or not G-CSF is a contributing factor is difficult to determine. On the other hand, it is a cautionary note as in other rare syndromes causing neutropenia, AML also has been documented in severe congenital neutropenia due to mutations, e.g., ELANE, HAX1 and in glycogen storage disease Ib.

\section{Discussion}

The pathophysiology of WHIM syndrome has been carefully investigated following the discovery of its causation by mutations in $C X C R 4$. As recently reviewed by Majumdar and Murphy, [12**] myelokathexis, i.e. abnormal retention of mature neutrophils in the bone marrow and recurrent infections, are the most common and determining features of this syndrome. The neutrophil abnormality is directly attributable to truncation in the cytoplasmic tail of the CXCR4 protein which normally mediates the internalization and down-regulation of this chemokine receptor. Normally when CXCL12 binds to CXCR4, neutrophils exit the marrow and enter the vascular compartment. The mutations prevent marrow to blood transit, leading to marrow accumulation of effete neutrophils. There is no simple explanation of lymphocytopenia in WHIMs. [12**] WHIM patients have mutant CXCR4 on their lymphocytes and markedly reduced circulating B and T cells. Levels of both $\mathrm{B}$ and $\mathrm{T}$ cells increased dramatically and in a parallel fashion when these patients are administered CXCR4 antagonists. $[14,15]$ The reservoir for $\mathrm{T}$ and $\mathrm{B}$ lymphocyte mobilization is not known but it is presumably the marrow, spleen and lymphoid tissues. The precise mechanisms underlying the narrow spectrum of infectious complications of this immunodeficiency syndrome is also unclear.

As revealed in this review and previous reports [1-11**], most infections are superficial, i.e. involve the skin and mucous membranes of the upper respiratory tract, vaginal mucosal surfaces and sometimes the perianal area. Deep abscesses in the skin occur because of a weak and delayed acute inflammatory response. The 24 patients in this report did not report frequent urinary tract infections and there were no episodes of bacteremia secondary to gastrointestinal infections. In our review, we found several episodes of pneumonia, but only one patient had appendicitis and one had cholecystitis. No patients died from sepsis, and hospitalizations occur very infrequently. Thus, the pattern of bacterial infections is distinctly different from patients with cyclic or severe congenital neutropenia; patients with similar neutrophil counts, but much more severe infections.

We are impressed by the morbidity of patients with recurrent otitis and significant hearing loss with damage to the tympanic membranes that is far worse than for many other type of neutropenia, making maintenance of normal hearing or potentially its improvement an objective for new therapies for WHIMs. Otherwise, WHIMs patients have a pattern of bacterial infections similar to patients with severe idiopathic neutropenia, a condition in which infections lead to a substantial but delayed neutrophilia and tissue neutrophil response.

We found lymphocytopenia in all patients, but variable patterns of warts. In our series, only a few patients had severe warts, but for these patients the warts cause severe disability. The

Curr Opin Hematol. Author manuscript; available in PMC 2021 January 01. 
determinants of the variability are unknown, but it is striking how some in a family can have severe warts and other family members living in the same household do not. This report mentions one case of severe, persistent molluscum contagiosum, a new viral infection complicating WHIMs which did not improve with G-CSF treatment. HPV associated malignancies involving the vagina, cervix and surrounding tissues and positive cervical exams are probably the most serious concerns for women with WHIMs.

Congenital malformations occur as part of WHIMs. In the 18 patients reported by Dotta et al, (14 females, 4 males in 15 families) there were 4 patients with congenital cardiovascular and renal anomalies. [11**] The anomalies were: tetralogy of Fallot, bicuspid aortic valve, pulmonary valve atresia, abnormal pulmonary arteries, agenesis of the second finger, unilateral radius hypoplasia, patent ductus arteriosus, pelvic dystrophy of the right kidney and retention of the testes. In the series of patients reported by Cohen et al, one patient had a double aortic arch and another had tetralogy of Fallot. In SCNIR series, 3 patients had congenital anomalies, one patent had ductus arteriosus, skin appendages with cartilaginous tissue and an anomalous kidney. A second patient had tetralogy of Fallot with dysplastic pulmonary artery and patent ductus arteriosus. A third patient had a more complex cardiac anomaly, pelvic kidney and malrotation of the intestines. The cardiac anomalies are similar to those seen in another type of congenital neutropenia, neutropenia associated with mutations in G6PC3, but the connection between these finding is not known. [20, 21]

This review reports long-term observational data for WHIMs treated with G-CSF. Through annual reports and regular contacts with patients, families and physicians, the SCNIR has learned that treatment of severe chronic neutropenia of diverse causes with G-CSF is best done by giving the lowest dose possible to achieve an ANC at the lower limit of normal and administering G-CSF daily or as frequently as possible to avoid adverse effects. For the 21or 24 patients reported herein, it was possible to adjust the dose and dose frequency to make GCSF treatment well tolerated in almost all patients and circumstances, and at the same time providing a consistent therapeutic benefit to reduce presumed bacterial infections of the skin and respiratory tract. WHIMs patients respond readily to G-CSF with typical transient increases in ANC. The inconsistencies in treatment are largely attributable to patient reluctance to do daily or alternate day subcutaneous injections.

\section{Conclusions}

Leukopenia, neutropenia and lymphocytopenia are the most consistent features of WHIM syndrome. Recurrent bronchitis and its complications, hearing loss from recurrent otitis media, disfiguring and disabling warts and HPV associated malignancies are its most serious complications. The variability of warts, respiratory and skin infections may be due to environmental factors or host defense mechanisms not yet identified. Some patients have congenital heart disease, most frequently tetralogy of Fallot. Mechanisms underlying the connection of CXCR4 mutations and the cardiac malformations in WHIMs are also unknown. The promise of novel therapies should lead to more frequent diagnosis and preventive treatment to avoid the numerous, serious complications of this syndrome, hopefully improving quality of life for these patients. [22**]

Curr Opin Hematol. Author manuscript; available in PMC 2021 January 01. 


\section{Acknowledgments}

Disclosure of Funding

This work was funded by the National Institutes of Health (NIH), National Institute of Allergy and Infectious Diseases (NIAID), Grant \#5R 24AI049393

\section{REFERENCES}

1. Ebrahim T, Hoolahan M, Donadieu J Review of case report literature highlights that WHIM syndrome has serious long-term outcomes beyond warts and infections. (Poster presentation) NORD Rare Diseases and Orphan Products Breakthrough Summit 102018.

2. Zuelzer WW. "Myelokathexis"--A new form of chronic granulocytopenia. Report of a case. N Engl J Med. 1964; 270: 699-704. [PubMed: 14101065] **The original report of WHIM syndrome

3. Krill CE Jr, Smith HD, Mauer AM. Chronic idiopathic granulocytopenia. N Engl J Med. 1964; 270:973-9. [PubMed: 14122792] * An interesting case report of the same patient as reported by Zeulzer

4. Hernandez PA, Gorlin RJ, Lukens JN, et al. Mutations in the chemokine receptor gene CXCR4 are associated with WHIM syndrome, a combined immunodeficiency disease. Nat Genet. 2003;34(1):70 [PubMed: 12692554] ** Report of the genetic cause for WHIMs

5. Hord JD, Whitlock JA, Gay JC, Lukens JN. Clinical features of myelokathexis and treatment with hematopoietic cytokines: a case report of two patients and review of the literature. J Pediatr Hematol Oncol. 1997; 19 (5):443-8. [PubMed: 9329467] Adaptive Immunodeficiency in WHIM Syndrome. Int J Mol Sci. 2018;20(1).

6. Gorlin RJ, Gelb B, Diaz GA, et al. WHIM syndrome, an autosomal dominant disorder: clinical, hematological, and molecular studies. Am J Med Genet. 2000; 91(5): 368-76. [PubMed: 10767001]

7. Beaussant Cohen S, Fenneteau O, Plouvier E, et al. Description and outcome of a cohort of 8 patients with WHIM syndrome from the French Severe Chronic Neutropenia Registry. Orphanet J Rare Dis. 2012; 7:71 [PubMed: 23009155] ** An important series of cases

8. Al Ustwani O, Kurzrock R, Wetzler M. Genetics on a WHIM. Br J Haematol. 2014;164(1):15-23. [PubMed: 24111611]

9. Badolato R, Donadieu J; WHIM Research Group. How I treat warts, hypogammaglobulinemia, infections, and myelokathexis syndrome. Blood. 2017;130(23):2491-2498. [PubMed: 29066537] * An important paper on therapy

10. Heusinkveld LE, Yim E, Yang A, et al. Pathogenesis, diagnosis and therapeutic strategies in WHIM syndrome immunodeficiency. Expert Opin Orphan Drugs. 2017;5 (10):813-825. [PubMed: 29057173]

11. Dotta L, Notarangelo LD, Moratto D, et al. Long-Term Outcome of WHIM Syndrome in 18 Patients: High Risk of Lung Disease and HPV-Related Malignancies. J Allergy Clin Immunol Pract. 2019;7(5):1568-1577. [PubMed: 30716504] ** An important series of cases describing the syndrome

12. Majumdar S, Murphy PM. Adaptive immunodeficiency in WHIM syndrome. Int J Mol Sci 2019;20(1).3.** Thorough report on the pathophysiology of WHIMs and it clinical manifestations

13. Aprikyan AG, Liles WC, Park JR, Jonas M, Chi EY, Dale DC. Myelokathexis, a congenital disorder of severe neutropenia characterized by accelerated apoptosis and defective expression of bcl-x in neutrophil precursors. Blood. 2000; 95:320-7. [PubMed: 10607719]

14. Dale DC, Bolyard AA, Kelley ML, et al. The CXCR4 antagonist plerixafor is a potential therapy for myelokathexis, WHIM syndrome. Blood. 2011;118(18):4963-6. [PubMed: 21835955]

15. McDermott DH, Liu Q, Ulrick J, et al. The CXCR4 antagonist plerixafor corrects panleukopenia in patients with WHIM syndrome. Blood. 2011;118(18):4957-62. [PubMed: 21890643]

16. McDermott DH, Liu Q, Velez D, et al. A phase 1 clinical trial of long-term, low-dose treatment of WHIM syndrome with the CXCR4 antagonist plerixafor. Blood. 2014;123(15):2308-16. [PubMed: 24523241] 
17. Dale DC, Firkin F, Bolyard AA, et al. Phase 2 study of X4P-001: A targeted oral therapy for patients with WHIM syndrome. (European Hematology Association [EHA] Meeting Abstracts). EHA Learning Center. 6 16, 2018; 215378.

18. McDermott DH, Pastrana DV, Calvo KR, et al. Plerixafor for the Treatment of WHIM Syndrome. N Engl J Med. 2019;380(2):163-170. [PubMed: 30625055] ** A preliminary report on outcomes for treatment of WHIMs with a CXCR4 antagonist

19. Dale DC, Bolyard AA, Schwinzer BG, Pracht G, Bonilla MA, Boxer L, Freedman MH, Donadieu J, Kannourakis G, Alter BP, Cham BP, Winkelstein J, Kinsey SE, Fier CJ, Zeidler C, Welte K. The Severe Chronic Neutropenia International Registry: 10-year follow-up report. Support Cancer Ther. 2006;3:220-31. [PubMed: 18632498]

20. Boztug K, Appaswamy G, Ashikov A, et al. A syndrome with congenital neutropenia and mutations in G6PC3. N Engl J Med. 2009;360(1):32-43. [PubMed: 19118303]

21. Banka S, Chervinsky E, Newman WG, Crow YJ, Yeganeh S, Yacobovich J, Donnai D, Shalev S. Further delineation of the phenotype of severe congenital neutropenia type 4 due to mutations in G6PC3. Eur J Hum Genet. 2011;19(1):18-22.

22. Heusinkveld LE, Yim E, Yang A, et al. Pathogenesis, diagnosis and therapeutic strategies in WHIM syndrome immunodeficiency. Expert Opin Orphan Drugs. 2017;5(10):813-825. [PubMed: 29057173 * An excellent review 


\section{Key Points}

- WHIMs is a rare immunodeficiency syndrome causing neutropenia and lymphocytopenia.

- WHIMs predisposes to bacterial and viral infections and HPV associated malignancies.

- Cardiac and renal anomalies are less common.

- $\quad$ CXCR4 antagonists are promising novel therapies. 


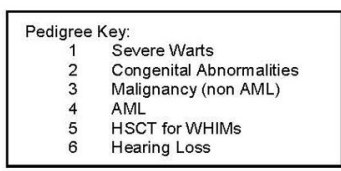

Pedigrees for 9 Families with WHIM Syndrome
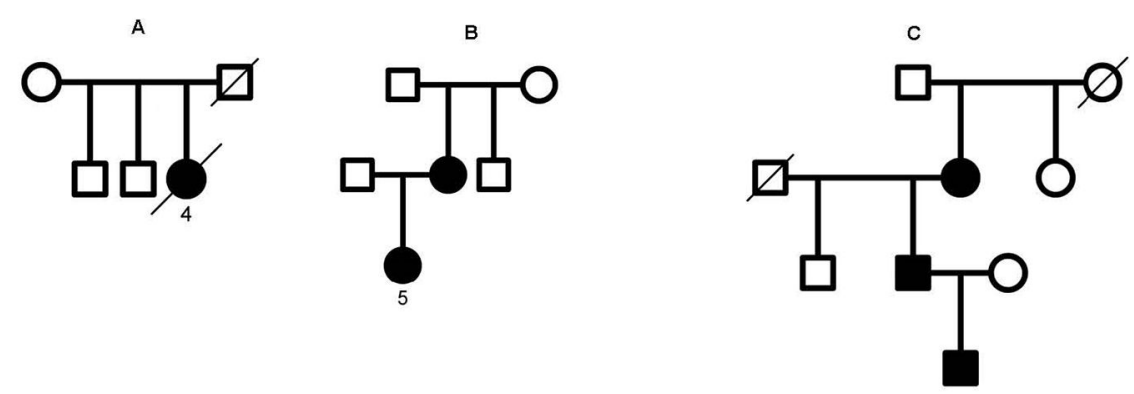

D
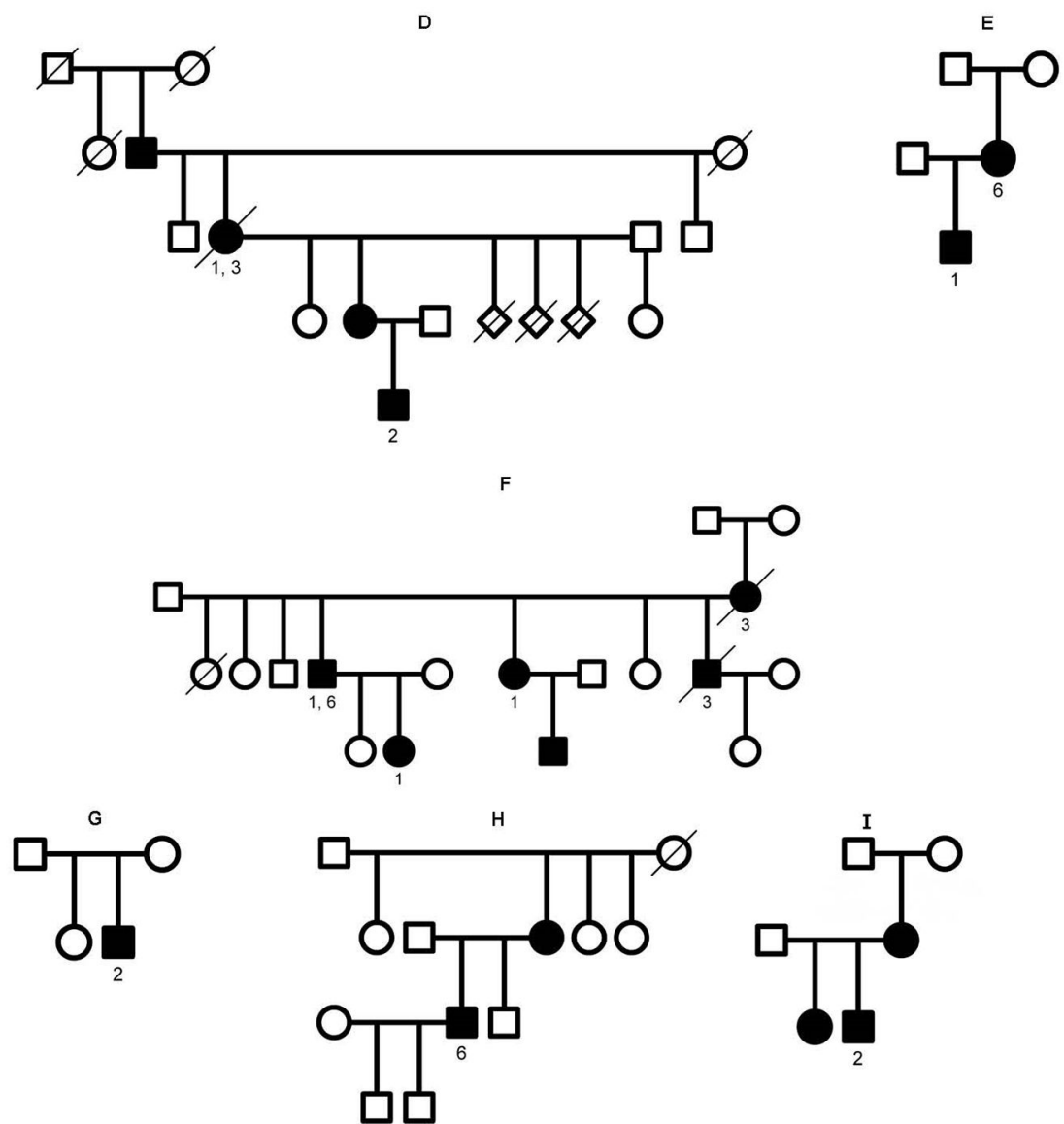

Figure 1 -.

Pedigrees for 9 Families with WHIM Syndrome 